\title{
The effect of density and diameter on haptic perception of rod length
}

\author{
TIN-CHEUNG CHAN \\ The Chinese University of Hong Kong, Shatin, New Territories, Hong Kong
}

\begin{abstract}
Three experiments on the effect of density and diameter on haptic perception of rod length are reported. In Experiment 1, the subjects wielded visually occluded rods of different densities. Perceived length was found to be affected by the density of the rod regardless of the actual length. In Experiment 2, three aluminum rods of different lengths with handles of four different diameters were wielded. Perceived length of the rod was found to be shorter as the diameter of the handle with which it was wielded increased. A diameter-length illusion was thereby produced. In Experiment 3, visually occluded rods of different diameters but of the same moment of inertia about the $x$-axis were wielded with the right hand, and tubes of different diameters were felt with the left hand. The subjects were instructed that their right hand was grasping a handle, and that the actual diameter of the rod could be felt with the left hand. Rods were perceived to be shorter if a larger diameter was felt with the left hand. The results showed that perceived length is not just a function of actual rod length, and that it is not accounted for by inertia only. The results are further discussed in terms of the nature of invariants and the effect of knowledge on perception.
\end{abstract}

It has been shown that the relative length of a visually occluded rod can be judged quite accurately by wielding it (Solomon \& Turvey, 1988). Such a judgment is claimed to demonstrate the information-perception specification, whereby perception is specific to information and information is specific to the environment (Gibson, 1979; Kugler \& Turvey, 1987). In vision, light reflected by the environment, called ambient light, carries with it the spatial and temporal patterning that can specify the layout of the environment. In the case of haptic perception of rod length, the changing flux of stimulation in the muscles and tendons in wielding the rod provides the patterning that specifies the length of the rod (Gibson, 1966). The length of the rod is not indicated by an image of length, but is specified by a force structure (not length) that produces tissue deformation on muscle and tendon. Moreover, it has been pointed out that because rod length is an enduring property of the environment, it has to be specified by an invariant, an enduring pattern that remains unchanged but that is revealed by changes in the stimulation. The invariant that specifies haptically perceived length has been found to be related to the moment of inertia - the resistance to rotational acceleration-experienced in wielding a rod.

Preparation of this article was supported by the Shaw College Student Campus Work Scheme (1993, fall) of the Chinese University of Hong Kong. The author would like to thank M. H. Bond for reading the first draft, and M. L. Braunstein, S. J. Lederman, and another anonymous reviewer for constructive criticism. Correspondence should be addressed to T.-C. Chan, Department of Psychology, The Chinese University of Hong Kong, Shatin, New Territories, Hong Kong (e-mail: tcchan@cuhk.hk).
To wield a rod about the $x$-axis with angular velocity $\omega_{1}$, an angular momentum $\mathbf{L}_{1}$ (see Appendix) is produced. If the motion is strictly along the $y z$ plane (Figure 1), it can be expressed by the equation $\mathbf{L}_{1}=I_{1} \omega_{1}$. The quantity $I_{1}$ is the moment of inertia of the rod about the $x$-axis. Similarly, $I_{2}$ is the moment of inertia about the $z$-axis, and $I_{3}$ is the moment of inertia about the $y$-axis. In three-dimensional rotation, the equation of motion, in its simplest form, consists of the three equations together. The three equations can be rewritten in matrix form as one equation, $\mathbf{L}=\mathbf{I} \omega$, where $\mathbf{I}$ is called an inertia tensor expressed in a $3 \times 3$ matrix (Goldstein, 1980). The three moments of inertia are the diagonal components of the diagonalized inertia tensor (see Appendix). It is these moments of inertia that have been found to comprise the invariant used in the judgment of rod length.

Even so, moment of inertia is not the flux of stimulation (proximal stimulus or haptic array), but a property of the grasped rod (distal stimulus). It should be the angular momentum (or the rate of change of angular momentum - the torque) and the velocity (or the rate of change of velocity - the acceleration) that produce the flux of stimulation in the muscles and joints.

What is the relationship between actual and perceived lengths, and what is the invariant force structure that specifies the perceived length? Since Solomon and Turvey's (1988) study, the first study of perceived length of a rod through rotation, there have been several claims. Solomon, Turvey, and Burton (1989), having shown that there must be a scaling function that maps the invariant onto a distance metric, claim, "It is noteworthy that the proposed scaling function yields an accurate estimate of reaching distance when the hand-held object is of uni- 


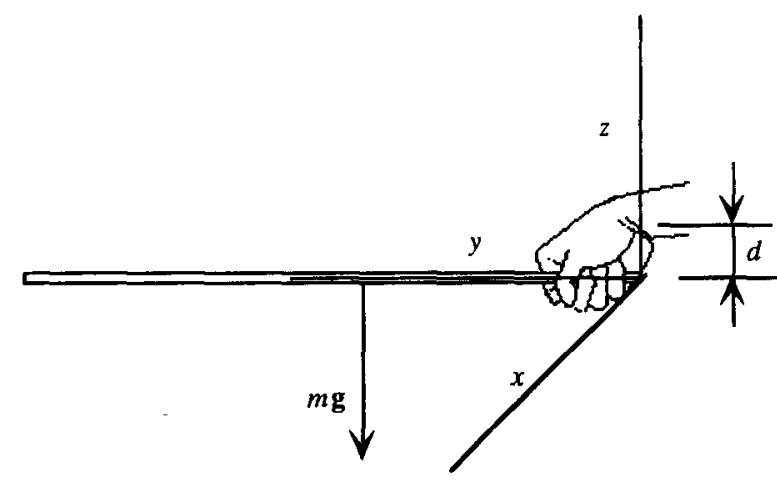

Figure 1. A hand-held rod is shown with respect to the coordinate system. The rod is grasped at one end, pivoted at the wrist. The weight $m$ g acts downward through the center of mass, and $d$ is the vertical distance of the wrist from the center of the rod.

form mass distribution" (p. 66). Similarly, Burton and Turvey (1991) claim that "when the hand-held object has a uniform density, perceived extents can match actual extent" (p. 129). Although Pagano and Turvey (1993) point out that actual length is not the same as perceived length, they assert that "perceived reachable distance was tied to I in a manner closely similar to actual reachable distance" (p. 147). It remains to be shown, however, whether this similarity between actual length and perceived length is an intrinsic relationship, or whether it just results from the way in which the rods were used in the previous experiments.

As to the identification of the invariant that specifies perceived length, Solomon et al. (1989) ascribe the picking up of the invariant to three successive operators: a differential operator that describes the motion of the hand-and-rod system; an integral operator that produces from the description of the motion the moment of inertia tensor; and an orthogonal operator that picks out the invariant from the inertia tensor. As to which elements in the inertia tensor are picked out as the invariant and scaled to the length of the rod, Solomon and Turvey (1988) assert that for slender rods, only the moment of inertia about the axis perpendicular to the rod has to be considered. It is claimed that "the dynamic behavior of the hand and rod system can be expressed in terms of a single $I$ " (p. 419) - that is, $I_{1}$, the moment of inertia about the $x$-axis (see Figure 1). Pagano and Turvey (1992) also make a similar assertion. Yet Pagano and Turvey (1993), by using a special rod with perpendicular branches, and by using multiple regression for the line of best fit, found the invariant to be made up of $I_{1}$ (the moment of inertia about the $x$-axis) and $I_{3}$ (the moment of inertia about the $y$-axis).

Three questions remain to be answered definitively: (1) Is the perceived length similar to the actual length? (2) Is $I_{3}$ actually involved in the perception of rod length? And (3) are other parameters involved besides the principal components of moment of inertia that would affect the haptic perception of rod length? The three experiments in the present study were aimed at answering these questions.

\section{EXPERIMENT 1 \\ Is the Perceived Length, $L_{\mathrm{p}}$, Similar to the Actual Length?}

That rod length is specified by the moment of inertia $I_{1}$ is incompatible with the claim that perceived length equals actual length. $I_{1}$ is affected by variables other than length, such as diameter and density (see Appendix). Pagano and Turvey (1992) state clearly that perceived length is mainly specified by the moment of inertia about the axis perpendicular to the rod length. Simple mechanics shows that moment of inertia increases with the density and thickness of a rod, variables that are unrelated to the actual length of the rod. Thus, logically, equality between perceived and actual lengths seems unlikely. Nor do empirical results support such a claim; indeed, in Solomon and Turvey's (1988) study, when solid aluminum rods were used, the subjects perceived the rods to be shorter than their actual lengths, but when denser solid steel rods were used, the perceived length was longer than the actual length. Although the difference in judgment is small compared with the difference in density, a difference is clear. In experiments conducted by myself, light, hollow metal rods were used, and the subjects underestimated rod length tremendously (Chan, 1994; Chan \& Turvey, 1991); therefore, perceived length could not be similar to the actual length of the rod.

To show that perceived length is not similar to the actual length, it is sufficient to show that perceived length is affected by density: For rods of the same length, then, denser rods will be perceived as longer. In the present experiment, four light, hollow steel rods and four solid aluminum rods were used. The rods were wielded behind an occluding board, and their lengths reported. For the same length of rod, the set of denser solid aluminum rods was expected to be perceived as longer.

\section{Method}

Subjects. Ten undergraduates in an introductory psychology course at the Chinese University of Hong Kong participated in partial fulfillment of a course requirement.

Apparatus. Four hollow homogeneous steel rods $(0.94-\mathrm{cm}$ diam) of $50,60,70$, and $80 \mathrm{~cm}$, with respective masses of 44.35 , $54.55,61$, and $70.5 \mathrm{~g}$, and four solid aluminum rods $(0.96-\mathrm{cm}$ diam) of $50,60,70$, and $80 \mathrm{~cm}$, with respective masses of 102.77 , $122.50,143.35$, and $164.62 \mathrm{~g}$, were used. The two sets of rods, with a .02-cm difference in diam, were regarded as equal in thickness. Two wooden boards $(1.5 \mathrm{~m}$ high $\times 1.2 \mathrm{~m}$ wide), with a vertical slit of $20 \mathrm{~cm}$ in between, were used as screens to occlude the hand and the grasped rods (Figure 2). A 0.8-m-high armrest (the back of a chair) was placed against the back of the slit. A piece of black cloth covered the top part of the slit. For the subjects reporting length, a length measurer that was $1.3 \mathrm{~m}$ in length was used. The length measurer had a sliding marker connected with a string mounted on a board. By pulling the string up or down at the starting edge of the board, the subjects could move the marker along a meter rule fixed at the top of the board. The perceived length of a rod could then be 


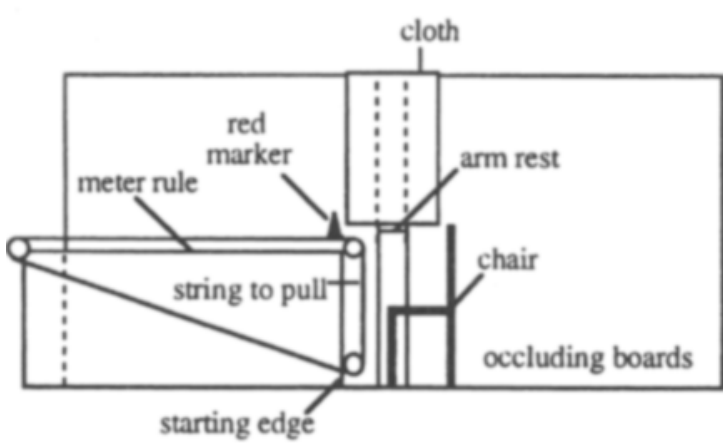

(A)

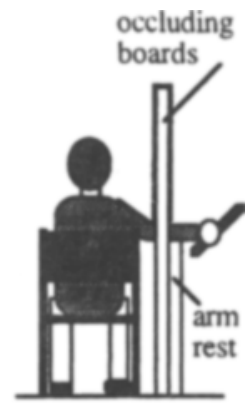

(B)

Figure 2. (A) Arrangement of the apparatus. (B) Diagram of a subject wielding a rod, with an arm inserted into the slot between the two occluding boards. The view of the length measurer as shown in $A$ is blocked by the subject.

translated to the distance of the marker from the starting edge. The length measurer was placed along the occluding board in front of the subject.

Procedure and Design. The subjects positioned their right arm on the armrest, with their hand behind the screens. Rods were placed by the experimenter into the right hand of each subject, and they were always gripped by the subjects so that the wrist was about $6 \mathrm{~cm}$ above the rod axis at the rear end (Figure 1). The subjects were told to grip the rods firmly, with the thumb positioned adjacent to the index finger, to ensure that they could not move the rod with the thumb independently of the wrist, which would have enabled the wrist to act as the pivot point of the rod-hand system. The subjects swung the rod up and down and circled the rod to perceive its length. In each trial, the subjects moved the marker away from themselves with their left hand to indicate the perceived length of the whole rod held in their right hand. They were encouraged to take as much time as necessary to perceive the rod length accurately. At the end of each trial, the rod was taken away and the subjects moved the marker back to the starting edge. Before the experimental trials, they were given a practice trial with a rod that was not used in the experiment.

In the experiment, the eight rods from both sets were presented randomly in a block of trials. There were four blocks, with a total of 32 trials. Perceived length of the whole rod was measured in each trial.

The experiment was a $2 \times 4$ (density of rod $\times$ rod length) within-subject design. Because perceived length of a rod is dependent on the moment of inertia of the rod about the $x$-axis, the density of a rod was expected to affect its perceived length.

Table 1

Perceived Lengths of the Two Sets of Rods in Experiment 1

\begin{tabular}{|c|c|c|c|}
\hline \multirow{2}{*}{$\begin{array}{l}\text { Rod Length } \\
\quad(\mathrm{cm})\end{array}$} & \multicolumn{2}{|c|}{$\begin{array}{l}\text { Moment of Inertia } \\
\left(\mathrm{g} \cdot \mathrm{cm}^{2} / 100\right)\end{array}$} & \multirow{2}{*}{$\begin{array}{l}\text { Perceived } \\
\text { Rod Length } \\
\text { (cm) }\end{array}$} \\
\hline & $I_{1}$ & $I_{3}$ & \\
\hline \multicolumn{4}{|c|}{ Hollow Steel Rod } \\
\hline 50 & 38.56 & 1.61 & 34.16 \\
\hline 60 & 67.42 & 1.98 & 39.50 \\
\hline 70 & 101.83 & 2.21 & 50.57 \\
\hline 80 & 152.94 & 2.55 & 57.91 \\
\hline \multicolumn{4}{|c|}{ Solid Aluminum Rod } \\
\hline 50 & 89.34 & 3.71 & 45.71 \\
\hline 60 & 151.41 & 4.42 & 60.40 \\
\hline 70 & 239.30 & 5.17 & 73.88 \\
\hline 80 & 357.12 & 5.94 & 82.08 \\
\hline
\end{tabular}

\section{Results and Discussion}

The means of the perceived forward length for different densities and actual lengths are listed in Table 1. An analysis of variance (ANOVA) showed that the effect of density was significant $[F(1,12)=78.84, p<.0001]$, indicating that density affects perceived length. The effect of length was significant $[F(3,36)=77.93, p<$ $.0001]$, indicating that length can be discerned. Together with the effect of density, these results showed that within each set of rod of equal density, longer rods are perceived as longer. There was an interaction between density and length $[F(3,36)=9.58, p<.0002]$, indicating a difference in the scaling functions of the actual-toperceived length between the two sets of rods (Figure 3). As shown in Figure 3, the regression coefficient $b$-weight for the lighter hollow steel rods was 0.82 , whereas that of the solid aluminum rods was 1.23. Regression of $\log$ $L_{\mathrm{p}}$ on $\log I_{1}$ (Figure 4 ) yielded an $R^{2}$ of .986 , indicating that when the thickness of the rod was kept constant, perceived length was mainly dependent on $I_{1}$, as previously claimed by Solomon and Turvey (1988) and Pagano and Turvey (1992).

Because $98.6 \%$ of the variance of perceived length is accountable by $I_{1}$, the effect of $I_{3}$ should be insignificant for length perception. Multiple regression of log perceived length on $\log I_{1}$ and $\log I_{3}$ yielded an $R^{2}$ of .987 , with partial $F$ s of $78.1(p=.0003)$ and $0.111(p=.75)$ for $\log I_{1}$ and $\log I_{3}$, respectively. $I_{3}$ was shown to be irrelevant to perceived length.

In sum, the results showed that because perceived length is mainly dependent on the first moment of inertia, perceived length is not similar to actual length if density is allowed to vary. Denser rods are perceived as longer.

\section{EXPERIMENT 2 \\ What Contributes to the Apparent Effect of $I_{3}$ ?}

In Experiment $1, I_{3}$ was shown to be irrelevant to the perceived length in wielding homogeneous rods of fixed thickness. In Pagano and Turvey's (1993) study, although perceived length was expressed as a function of 


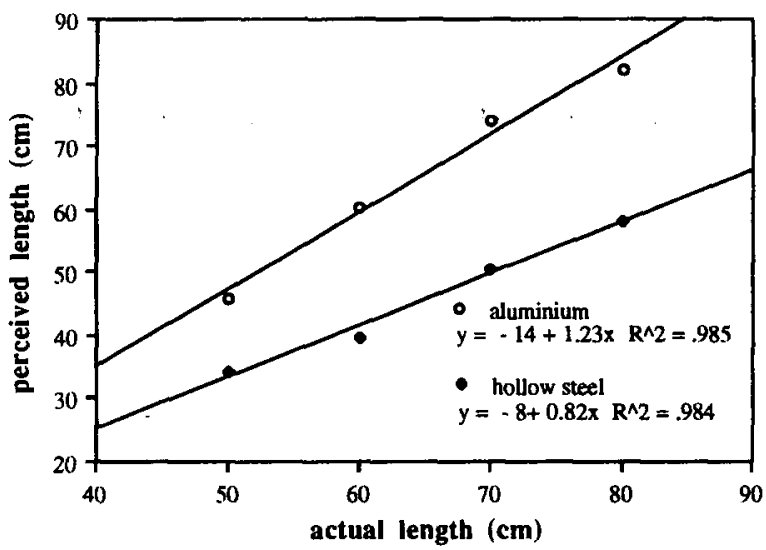

Figure 3. The scaling function of actual-to-perceived length is different for rods of different densities (Experiment 1).

$I_{1}$ and $I_{3}$ (perceived length $=389 I_{1}^{42} I_{3}^{-.01}$ ), the effect of $I_{3}$ was not significant. However, in Fitzpatrick, Carello, and Turvey's (1994) study, $I_{3}$ was significant. Even so, three different equations were found in three experiments when wielding homogeneous rods with or without handles. With rods of different diameters and lengths, but of the same material, perceived length was found to be $4.74 I_{1}^{0.36} I_{3}^{-0.25}$. With rods made of materials of different densities inserted in handles of constant thickness, perceived length was found to be $2.15 I_{1}^{0.37} I_{3}^{-0.17}$. With lengths of rods of the same material, but attached to handles of different diameters, perceived length was found to be $1.03 I_{1}^{0.54} I_{3}^{-0.34}$. In fact, with the data listed, in the first and second experiments, $I_{1}$ was highly correlated with $I_{3}$, a condition of collinearity in which multiple regression should not be conducted. In addition, $I_{3}$ was not significant in the second experiment.

The effect of $I_{3}$ on perceived length was shown to be insignificant in the present Experiment 1. It is uncertain as to what the apparent effect of $I_{3}$ is really attributed by

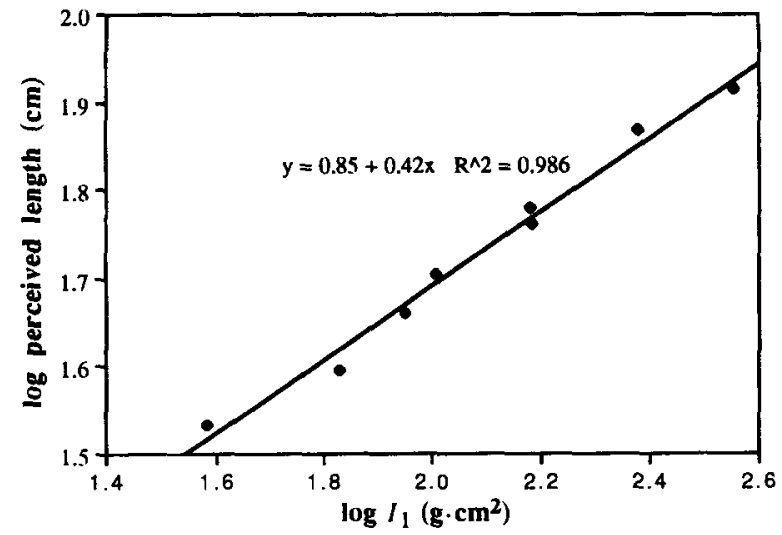

Figure 4. For rods with similar thickness, perceived length is mainly accounted for by the moment of inertia about the axis perpendicular to the rod, $I_{1}$ (Experiment 1).
Fitzpatrick et al. (1994). In Pagano and Turvey's (1993) study, with the diameter of the rod held constant and $I_{3}$ systematically varied with $I_{1}$ such that $I_{1}$ and $I_{3}$ were not correlated, the effect of $I_{3}$ was still insignificant. A review of Fitzpatrick et al.'s (1994) two experiments, in which $I_{3}$ was significant, shows that, in both cases, the diameter of the rod was varied. It is suspected that the apparent effect of $I_{3}$ was due to its correlation with the diameter, $d$, which really affected the perceived length. In fact, a multiple regression of average $\log L_{\mathrm{p}}$ on $\log I_{1}$ and $\log d$ in the third experiment yielded a significant $R^{2}$ of .98 , with partial $F$ s of $133.52(p=.0001)$ for $\log I_{1}$ and $127.46(p=.0001)$ for $\log d$. There are reasons why $I_{3}$ could not be an appropriate predictor. In the previous experiments, the calculated power coefficient for $I_{3}$ fluctuated greatly. The effect of $I_{3}$ was significant only for a few specific conditions. Moreover, an increase of actual length in homogeneous rods should be concomitant with an increase of $I_{3}$, and yet the power coefficient of $I_{3}$ in perceived length was negative.

To show that perceived rod length is affected by the diameter of the rod rather than by $I_{3}$, handles of different diameters but of similar $I_{3}$ can be used. An unreported pilot experiment (Chan, 1990) showed that when identical rods were wrapped with different thicknesses of cardboard paper as handles, rods with handles of thicker diameters were perceived as shorter. Because the paper wrapping was light, both of the changes in $I_{1}$ and $I_{3}$ produced by the paper wrapping were too small to affect the perceived length. Moreover, the distance of the wrist from the axis of the rod ( $d$ in Figure 1) with increasing thickness of the wrapping did not change. The shortening of the perceived length could only be a result of the change in the diameter of the paper handle.

In this experiment, four aluminum handles of different diameters were used with three lengths of aluminum rod. The four handles were hollowed so that the difference in mass, and thus the difference in the moment of inertia, was kept to a minimum. With handles of larger diameters, the perceived length was expected to be shorter.

\section{Method}

Subjects. Ten undergraduates in an introductory psychology course at the Chinese University of Hong Kong participated in partial fulfillment of a course requirement.

Apparatus. Three solid aluminum rods $(0.96-\mathrm{cm}$ diam $), 60,70$, and $80 \mathrm{~cm}$ in length and with respective masses of $122.5,143.4$, and $164.7 \mathrm{~g}$, and four hollowed handles (12 cm long), 2, 3, 4, and $5 \mathrm{~cm}$ in diam and with respective masses of $73.3,115.7,115.5$, and $115.5 \mathrm{~g}$, were used. In each trial, one of the handles was fixed to the end of each rod (Figure 5). The occluding board and the length measurer were the same as those used in Experiment 1.

Procedure and Design. The subjects wielded the occluded rod to judge the length of the rod as in Experiment 1, except that in each trial, handles with the rod attached were placed into their right hand. The handles were grasped such that the wrist was about $6 \mathrm{~cm}$ above the axis at the rear end. The subjects wielded the rod with their right hand and reproduced the perceived whole length of the rod on the length measurer with their left hand. They were told 


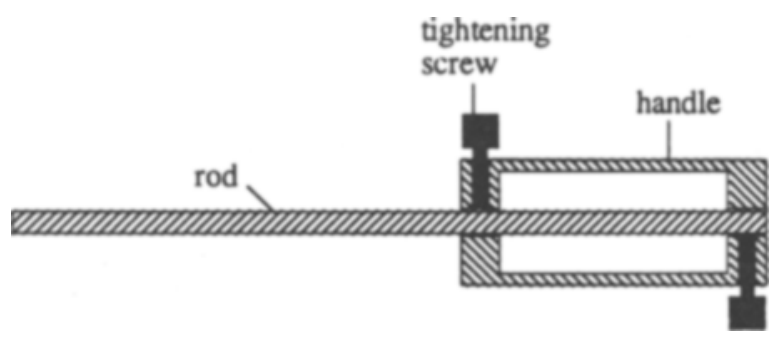

Figure 5. Cross-section to show the fitting of a rod into a handle (Experiment 2).

that what they grasped was a handle fixed to the end of each rod, and that all handles were of the same length and were only slightly longer than the width of their palm.

The 12 rod-handle combinations were presented randomly in a block of trials. There were four blocks, with a total of 48 trials. Perceived length of the whole rod was measured in each trial.

This experiment was a $4 \times 3$ (handle $\times$ rod length) within-subject design. It was expected that the diameter of the handle would affect the perceived rod length.

\section{Results and Discussion}

The means of the perceived rod length for different handle thicknesses and moments of inertia are listed in Table 2. An ANOVA on mean perceived rod length showed that the effect of handle thickness was significant $[F(3,27)$ $=42.1, p<.0001]$. Together with the mean perceived length, the results showed that rods with thicker handles were perceived as shorter. The effect of length was significant $[F(2,27)=48.77, p<.0001]$, indicating that rod length can be discerned. There was a marginal interaction between handle thickness and length $[F(6,54)=$ $2.28, p<.05]$.

To find out whether $I_{3}$ and $I_{1}$ can be used together in the multiple regression equation $\log I_{\mathrm{p}}=a \log I_{1}+$ $b \log I_{3}+\log c\left(\mathrm{a} \log\right.$ transformation of the equation $I_{\mathrm{p}}=$ $\left.c l_{1}^{\mathrm{a}} I_{3}^{\mathrm{b}}\right)$, correlations between $\log I_{1}, \log I_{3}$, and $\log d(\mathrm{di}-$ ameter of the rod) were found. The correlation between $\log I_{1}$ and $\log I_{3}$ was .579 , between $\log I_{1}$ and $\log d$ it was 0 , and between $\log I_{3}$ and $\log d$ it was .78. Because of collinearity, it is inappropriate to regress $\log L_{\mathrm{p}}$ on $\log I_{1}$

Table 2

Perceived Lengths of the Three Rods With Different Handle Thicknesses

\begin{tabular}{ccccc}
\hline $\begin{array}{c}\text { Handle } \\
\text { Diameter } \\
(\mathrm{cm})\end{array}$ & $\begin{array}{c}\text { Rod } \\
\text { Length } \\
(\mathrm{cm})\end{array}$ & \multicolumn{2}{c}{ Total $\left(\mathrm{g} \cdot \mathrm{cm}^{2}\right) / 10^{4}$} & $\begin{array}{c}\text { Perceived } \\
\text { Length } \\
(\mathrm{cm})\end{array}$ \\
\hline 2 & 60 & 15.80 & 0.73 & 21.73 \\
& 70 & 24.60 & 0.81 & 26.87 \\
3 & 80 & 36.39 & 0.88 & 32.75 \\
& 60 & 16.22 & 0.90 & 17.87 \\
& 70 & 25.02 & 0.97 & 24.66 \\
4 & 80 & 36.81 & 1.05 & 28.37 \\
& 60 & 16.25 & 0.93 & 16.91 \\
& 70 & 25.05 & 1.00 & 22.33 \\
5 & 80 & 36.84 & 1.08 & 24.82 \\
& 60 & 16.27 & 0.96 & 15.37 \\
& 70 & 25.06 & 1.04 & 19.62 \\
& 80 & 36.85 & 1.11 & 23.74 \\
\hline
\end{tabular}

and $\log L_{3}$; yet $\log I_{1}$ and $\log d$ can be used in the same equation.

Regression of $\log L_{\mathrm{p}}$ on $\log I_{1}$ alone yielded an $R^{2}$ of only .64 $\left(L_{\mathrm{p}}=0.042 I_{1}^{0.51}\right)$. The variance in $L_{\mathrm{p}}$ introduced by changing $d$ is orthogonal to the variance introduced by changing $I_{1}$ (Figure 6). It is this variance introduced by changing $d$ that reduces the $R^{2}$ from .986 in Experiment 1 to 64 . Multiple regression of $\log L_{\mathrm{p}}$ on $\log$ $I_{1}$ and $\log d$ yielded an $R^{2}$ of $.98[F(2,9)=244.04, p<$ .0002 ], with partial $F \mathrm{~s}$ of $327.463(p<.0002)$ and $170.88(p<.0002)$ for $\log I_{1}$ and $\log I_{3}$, respectively $\left(L_{\mathrm{p}}=.058 I_{1}^{0.52} d^{-0.18}\right)$. Because $\log L_{\mathrm{p}}$ correlated negatively with $\log d(r=-0.57)$, a negative power coefficient is appropriate.

Why does diameter give a shrinking illusory effect on perceived rod length? This question can be addressed by referring to the study of Armstrong and Chaffin (1978), who show that a specific degree of joint rotation of the fingers corresponds to a specific amount of tendon displacement at the wrist. When the fingers are stretched out, as when holding a larger object, there is greater tendon displacement, resulting in greater muscle elongation and, thus, greater wrist stiffness. This is compatible with the findings of Davis and Brickett (1977) that indicate that in preparing to lift a larger can, greater muscle tension is produced, resulting in the size-weight illusion. These findings suggest that with increased wrist stiffness and muscle tension in preparing to lift a rod of larger diameter, the experienced moment of inertia in wielding the rod would be reduced, resulting in shorter perceived length.

Ellis and Lederman (1993) report similar findings of a size-weight illusion in holding objects, and claim that a haptic volume cue is sufficient to produce such an illusion. Lederman, Ganeshan, and Ellis (in press), using horizontal rods of different diameters held stationary, show that subjects perceived rods of larger diameters as lighter and shorter simultaneously. It is thus suggested that the diameter-length illusion may be mediated by a weight percept. In other words, the subjects used perceived weight to judge rod length. Since rods of larger diameters were perceived as lighter, they were also perceived as shorter. This weight is defined as a function of the gravitational pull (force) on the rod and the torque produced by the rod on the grasping hand. It is the referred weight rather than the actual weight of the rod, and it can vary even though the actual weight remains constant. Though such an explanation is possible, it is not yet certain, in holding rods stationary, whether perceived length is mediated through the referred weight, which is a function of the torque felt in holding the rod, or whether it is affected directly by the felt torque. Further experimentation may have to be conducted for clarification.

Nevertheless, results in this area of research show very clearly that in wielding rods of identical moment of inertia $I_{1}$, rods with greater diameters would be perceived as shorter. Let us call this factor the muscular constraint. In addition, when a rod is held, the diameter 


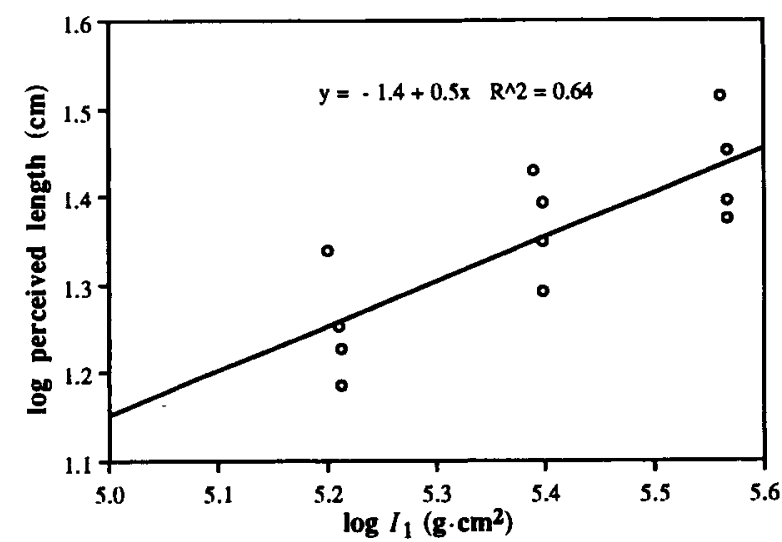

Figure 6. With variation of rod thickness, an additional variance of perceived length orthogonal to the variance produced by $I_{1}$ is produced (Experiment 2).

of the rod is known. The known diameter is purely geometric in nature, and may have an effect on perceived length. To differentiate the effect of the grasped diameter from the effect of the known diameter, let us call the latter effect the geometric constraint. To investigate whether knowing the diameter of a rod actually has an effect on the perceived length, the following experiment was conducted.

\section{EXPERIMENT 3 \\ The Effect of Perceived Diameter on Perceived Rod Length}

To separate the muscular constraint from the geometric constraint, in this experiment, information about the diameter of the rod was felt by the left hand independently of the right hand, which wielded the rod. Three rods of equal $I_{1}$ but of different diameters were wielded with the right hand behind an occluding board and their perceived lengths reported. The subjects were informed that what they were holding was just the handle, which was about the length of their palm, while the actual diameter of the rod could be felt with the left hand. Plastic tubes of three different diameters were felt by subjects using their left hand. It was expected that wrist stiffness (muscular constraint) and knowledge of the diameter (geometric constraint) would affect perceived rod length independently. Greater diameters felt with the left hand would make the perceived length of the rod wielded in the right hand shorter.

\section{Method}

Subjects. Eight undergraduates in an introductory psychology course at the Chinese University of Hong Kong participated in partial fulfillment of a course requirement.

Apparatus. Three hollow homogeneous metal rods of different thickness and length, but of equal principle moment of inertia about the $x$-axis, $I_{1}$ (gripped at $5 \mathrm{~cm}$ from one end), were wielded with the right hand. Detailed specifications of the three rods are shown in Table 3. Three plastic tubes of $1.3,2.2$, and $3.77 \mathrm{~cm}$ diam were felt by the left hand. The occluding board and the length measurer were the same as those used in Experiment 1.

Procedure and Design. The subjects wielded the occluded rod to judge the length of the rod as in Experiment 1, except that in each trial, while they wielded the rod grasped with the right hand, one of the three plastic tubes of different diameters was presented in random order to be felt by the left hand. The subjects were instructed that what they were grasping with their right hand was the handle of a rod, and that the diameter of the rod could be felt with their left hand. They were also instructed not to use logical deduction, but to perceive the length directly and intuitively. The experimenter held on to the plastic tube so that the subjects could not sense the weight of the tube. After perceiving the length of the metal rod, while still wielding the metal rod with their right hand, the subjects reported perceived length by adjusting the length measurer with the left hand. There were nine combinations of the three metal rods with the three plastic tubes, presented randomly in a block of trials. There were four blocks of trials, with a total of 32 trials. Before the experimental trials, the subjects were given a practice trial with a rod that was not used in the experiment.

Table 3

Perceived Lengths of the Three Rods With Identical Moment of Inertia $I_{1}$, but With Rods of Different Diameters Felt With the Left Hand

\begin{tabular}{|c|c|c|c|c|c|}
\hline \multicolumn{4}{|c|}{ Dimensions of Rods Wielded } & \multirow{2}{*}{$\begin{array}{l}\text { Diameter of } \\
\text { Rods Felt with } \\
\text { Left Hand } \\
\text { (cm) }\end{array}$} & \multirow{2}{*}{$\begin{array}{c}\text { Perceived Length } \\
\text { of Rod } \\
(\mathrm{cm})\end{array}$} \\
\hline Length $(\mathrm{cm})$ & Mass (gm) & Diameter $(\mathrm{cm})$ & $I_{1}\left(\mathrm{~g} \cdot \mathrm{cm}^{2}\right) / 10^{4}$ & & \\
\hline \multicolumn{6}{|c|}{ Thick } \\
\hline 40.5 & 313.0 & 2.50 & 12.71 & $\begin{array}{l}3.77 \\
2.21 \\
1.31\end{array}$ & $\begin{array}{l}34.45 \\
40.77 \\
43.58\end{array}$ \\
\hline \multicolumn{6}{|c|}{ Medium } \\
\hline 68.5 & 99.0 & 1.25 & 12.70 & $\begin{array}{l}3.77 \\
2.21 \\
1.31\end{array}$ & $\begin{array}{l}41.80 \\
47.78 \\
53.99\end{array}$ \\
\hline \multicolumn{6}{|c|}{ Thin } \\
\hline 80.0 & 69.5 & 1.00 & 12.70 & $\begin{array}{l}3.77 \\
2.21 \\
1.31\end{array}$ & $\begin{array}{l}48.76 \\
52.53 \\
57.47\end{array}$ \\
\hline
\end{tabular}

Note-The rods were all grasped with the wrist at $5 \mathrm{~cm}$ from the rear end. The moments of inertia of the three rods are roughly the same. 


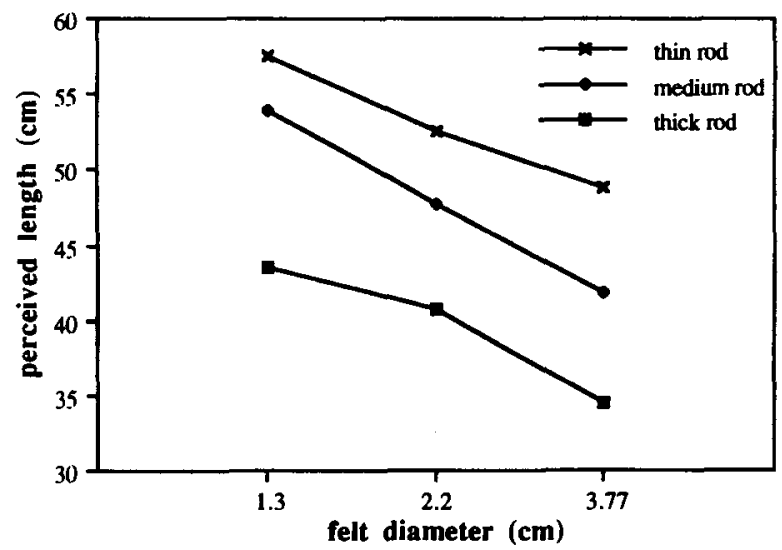

Figure 7. Perceived length is affected by the grasped diameter and the knowledge of the diameter of the rod (Experiment 3).

The experiment was a $3 \times 3$ (felt diameter $\times$ wielded rod) within-subject design. Both variables were of fixed effect. It was predicted that with the three metal rods of equal $I_{1}$, rods of greater diameter would be perceived as shorter. It was also predicted that when plastic tubes of greater diameter were felt with the left hand, the rods wielded in the right hand would be perceived as shorter.

\section{Results and Discussion}

The means of perceived length are plotted in Figure 7. An ANOVA showed that the effect of diameter of the wielded rod was significant $[F(2,14)=9.637, p<.0025]$. This can be explained by the wrist-stiffening effect with handles of larger diameter. Further, the effect of the diameter of the plastic tubes felt by the left hand of the subjects was also significant $[F(2,14)=14.654, p<.0005]$. This must be explained in terms of knowledge of the diameter of the rod held. No interaction was shown $[F(2,28)<1]$, indicating that the two effects are orthogonal.

These results clearly indicate that haptic perception of rod length depends not on inertia tensor alone, but on two additional orthogonal variables that were revealed by using rods of different diameters - namely, muscular and geometric constraints. Different constraints can be applied to different parts of the body for a single perception.

\section{GENERAL DISCUSSION}

In summary, the results of Experiment 1 showed that in wielding cylindrical rods of similar thickness made from different materials, actual length is not similar to perceived length. Denser rods are perceived as longer, depending on the magnitude of I1. I3 is not involved in the perception of the whole rod length. The results of Experiment 2 showed that the thickness of handle has an effect on perceived length: Perceived length is reduced when grasping rods of greater diameters. This can be explained by an increase in wrist stiffness when grasping a handle of greater diameter, producing a diameter-length illusion similar to the size-weight illusion. Yet there may be another interrelated component-the knowledge of the diameter of the handle or the rod which also affects perceived length. The latter component was separated out in Experiment 3. When the rod wielded in the right hand was implied to be greater in diameter, the length was perceived to be shorter. These results have important implications as far as two issues are concerned - the nature of invariants in haptic perception of rod length, and the effect of knowledge on perception.

\section{The Nature of Invariants in Haptic Perception of Rod Length}

An invariant is the consistent pattern of stimulation that specifies a perceived property such as length (Gibson, 1979; Turvey, Solomon, \& Burton, 1989). The aim of the present research, in concert with the previous work by other researchers, is to find out which is the invariant that actually specifies perceived length in the haptic sense. The invariant is expected to be a covariation of several physical variables giving rise to perceived lengths close to actual lengths. Perceived length has been shown to be proportional to the variable $I_{1}$ (Solomon \& Turvey, 1988), which increases with density and thickness in a way that is unrelated to the actual length of the rod. In order that perceived length is similar to actual length, there must be a covariable to reduce the effect of $I_{1}$ due to density and thickness. $I_{3}$ is the best candidate, and it has indeed been picked by previous researchers as the covariant of $I_{1}$ (Fitzpatrick et al., 1994; Pagano \& Turvey, 1992). Nevertheless, there is no convincing empirical evidence that $I_{3}$ is a covariant of $I_{1}$ for perception of rod length.

Experiments 2 and 3 showed, however, that $d$, the diameter of the handle, is a covariant of $I_{1}$ in the haptic perception of rod length. Such covariation would constrain perceived length to its actual magnitude with thicker rods of the same material. It is interesting that there are two independent factors to constrain the perceived length due to variation in thickness, as revealed in Experiments 2 and 3-namely, wrist stiffness and knowledge of rod thickness. Such covariation cannot, however, eliminate the variation due to the density of the material of the rod. Thus, with denser rods, perceived length is longer, as shown in Experiment 1.

Thus, unlike the perception of looming (Lee, 1976), where a specific invariant specifies the time-to-contact, in haptic perception of rod length, there is no invariant that specifies perceived length that is identical to actual length; evolution does not provide humans with an invariant to specify length haptically with accuracy. It seems that it is primarily the functions of knowledge and practice that compensate for such inadequacy.

\section{The Effect of Knowledge on Perception}

In Experiment 3, the diameter felt with the left hand altered the perceived length of the rod wielded in the right hand. The results indicated that variables experienced with different parts of the body can be combined to specify one single rod length. Such integration has been demonstrated in a study by Carello, Fitzpatrick, Domaniewicz, Chan, and Turvey (1992), in which sub- 
jects perceived the length of rods held at one end with a downward thrust on the leg and an upward thrust on the finger. The feeling of the diameter of the rod in the present Experiment 3, however, has a unique characteristic: It is independent of the wielding of the rod by the subject with his or her right hand. Indeed, such a presentation can be replaced by a statement or a picture. Buck (1990) called such statements descriptive knowledge. The results showed that such knowledge modifies the perceived length of a rod.

Knowledge has been shown to affect perception in various modalities: Lindauer (1989) has shown that expectation alters ambiguous figure-ground perception; Van der Velde, Van der Heijden, and Schreuder (1989) have shown that knowledge affects visual word perception in context-dependent migration; Elman and McClelland (1988) have shown that knowledge in English affects speech perception with lexically restored phonemes; and Appelle and Countryman (1986) have shown that knowledge of the standard would reduce the haptic oblique effect. It appears that any theory in perception must be able to account for the effect of knowledge on perception.

Traditionally, the notion that knowledge is required for perception would mean that with sensory inputs impinging on the sensory receptors, specific memories have to be retrieved from storage to fuse with the sensory input. According to this view, perceptual knowledge is not primary. We can only understand what we see now in terms of the past.

Gibson (1979) rejects such a dichotomy between the past and the present (see also Turvey \& Shaw, 1979; Turvey, Shaw, Reed, \& Mace, 1981), and instead regards information as coming in a stream. What is available visually with one saccade at any putative moment is only a fraction of a surface that happens to be in the field of vision. This snapshot is not taken as the basis of perception. Gibson (1979) suggests that the perceptual system has an input-output loop such that invariants can be detected over time, and the invariant, once detected, will be available for succeeding processes. Invariants detected later can be integrated with previous invariants. Without the dichotomy between past and present, the extraction of invariants has no boundaries. According to this view, knowledge is the extraction of invariants independently of the stimulus flux. Conception is an extension of perception (Turvey et al., 1989). To perceive the environment and to conceive it are different in degree but not in kind; one is continuous with the other.

The results of the present research show that in haptic perception of rod length, there is no physical invariant that specifies the actual length. Perception of the length of a rod when wielded is affected by moment of inertia as a physical invariant, by grasped diameter as a muscular constraint, and by known diameter as a geometric constraint. According to the view presented above, the muscular and geometric constraints can be considered as supplementary constraints, and perception due to detection of a physical invariant is modified by various constraints. Such an explanation, I believe, is consistent with the ecological view of perception.

\section{REFERENCES}

Appelle, S., \& Countryman, M. (1986). Eliminating the haptic oblique effect: Influence of scanning incongruity and prior knowledge of the standards. Perception, 15, 325-329.

ARMSTRONG, T. J., \& CHAFFIN, D. B. (1978). An investigation of the relationship between displacement of the finger and wrist joints and the extrinsic finger flexor tendons. Journal of Biomechanics, 11, 119-128.

Buck, R. (1990). William James, the nature of knowledge, and current issues in emotion, cognition, and communication. Personality \& Social Psychology Bulletin, 16, 612-625.

Burton, G., \& Turvey, M. T. (1991). Attentionally splitting the mass distribution of hand-held rods. Perception \& Psychophysics, 50, 129-140.

Carello, C., Fitzpatrick, P., Domaniewicz, I., Chan, T.-C., \& Turvey, M. T. (1992). Effortful touch with minimal movement. Journal of Experimental Psychology: Human Perception \& Performance, 18, 290-302.

ChAN, T.-C. (1990). [The role of diameter of the handle on the perception of rod length]. Unpublished raw data.

Chan, T.-C. (1994). Haptic perception of partial-rod lengths with the rod held stationary or wielded. Perception \& Psychophysics, 55, $551-561$

Chan, T.-C., \& Turvey, M. T. (1991). Perceiving the vertical distances of surfaces by means of a hand-held probe. Journal of Experimental Psychology: Human Perception \& Performance, 17, 347-358.

DAvis, C. M., \& BRICKETT, P. (1977). The role of preparatory muscular tension in the size-weight illusion. Perception \& Psychophysics, 22, 262-264.

ELLIS, R. R., \& LEDERMAN, S. J. (1993). The role of haptic versus visual volume cues in the size-weight illusion. Perception \& Psychophysics, 53, 315-324.

Elman, J. L., \& MCClelland, J. L. (1988). Cognitive penetration of the mechanisms of perception: Compensation for coarticulation of lexically restored phonemes. Journal of Memory \& Language, 27, 143-165.

FitzPatrick, P., Carello, C., \& Turvey, M. T. (1994). Eigenvalues of the inertia tensor and exteroception by the "muscular sense." Neuroscience, 60, 551-568.

GiBSON, J. J. (1966). The senses considered as perceptual systems. Boston, MA: Houghton Mifflin.

Gibson, J. J. (1979). The ecological approach to visual perception. Boston, MA: Houghton Mifflin

Goldstern, H. (1980). Classical mechanics. Reading, MA: AddisonWesley.

Hosington, L. B. (1920). On the non-visual perception of the length of lifted rods. American Journal of Psychology, 31, 144-146.

KUGLER, P. N., \& TURVEY, M. T. (1987). Information, natural law, and the self-assembly of rhythmic movement. Hillsdale, $\mathrm{NJ}$ : Eribaum.

Lederman, S. J., Ganeshan, S. R., \& Ellis, R. E. (in press). Haptic perception of the extent of statically held rods. Journal of Experimental Psychology: Human Perception \& Performance.

LEE, D. (1976). A theory of visual control of braking based on information about time-to-collision. Perception, 5, 437-459.

LiNDAUER, M. S. (1989). Expectation and satiation accounts of ambiguous figure-ground perception. Bulletin of the Psychonomic Society, 27, 227-230.

Pagano, C. C., \& Turvey, M. T. (1992). Eigenvectors of the inertia tensor and perceiving the orientation of a hand-held object by dynamic touch. Perception \& Psychophysics, 52, 617-624.

Pagano, C. C., \& Turvey, M. T. (1993). Perceiving by dynamic touch the distances reachable with irregular objects. Ecological Psychology, 5, 125-151.

Solomon, H. Y., \& TuRvey, M. T. (1988). Haptically perceiving the distances reachable with hand-held objects. Journal of Experimental Psychology: Human Perception \& Performance, 14, 404-427.

Solomon, H. Y., Turvey, M. T., \& Burton, G. (1989). Perceiving ex- 
tents of rods by wielding: Haptic diagonalization and decomposition of the inertia tensor. Journal of Experimental Psychology: Human Perception \& Performance, 15, 58-68.

Turvey, M. T., \& Shaw, R. E. (1979). The primacy of perceiving: An ecological reformulation of perception for understanding memory. In L. G. Nilsson (Ed.), Perspectives on Memory Research: Essays in Honor of Uppsala University's 500th Anniversary (pp. 167-222). Hillsdale, NJ: Erlbaum.

Turvey, M. T., Shaw, R. E., Reed, E. S., \& Mace, W. M. (1981). Eco- logical laws of perceiving and acting: In reply to Fodor and Pylyshyn (1981). Cognition, 9, 237-304.

Turvey, M. T., Solomon, H. Y., \& Burton, G. (1989). An ecological analysis of knowing by wielding. Journal of the Experimental Analysis of Behavior, 52, 387-407.

VAN DER Velde, F., van der Heidden, A. H., \& SChreuder, R. (1989). Context-dependent migrations in visual word perception. Journal of Experimental Psychology: Human Perception \& Performance, 15, 133-141.

\section{APPENDIX}

Dynamic Variables Used in This Article

\begin{tabular}{lc} 
Variables with Definitions and Formulae & Units \\
\hline Angular velocity $(\omega)$ : The velocity of rotation. & $\mathrm{rad} / \mathrm{sec}$ \\
$\omega=\mathbf{v} / r$, where $r$ is the radius of rotation and $\mathbf{v}$ is the linear velocity. & $\mathrm{g} \cdot \mathrm{cm}^{2} / \mathrm{sec}$
\end{tabular}

$\mathbf{L}=r \times \mathbf{p}$, where $r$ is the radius of the trajectory, $\mathbf{p}$ is the linear momentum, and $\times$ is the cross product for vector multiplication.

Inertia tensor (I): A property of the rod that characterizes the resistance of rotation of the rod in three dimensions; it is represented by a $3 \times 3$ matrix.

$\mathrm{g} \cdot \mathrm{cm}^{2}$

In its simplest form, it is:

$$
\left[\begin{array}{ccc}
I_{1} & 0 & 0 \\
0 & I_{2} & 0 \\
0 & 0 & I_{3}
\end{array}\right]
$$

The equation $\mathbf{L}=\mathbf{I} \omega$ is equivalent to:

$$
\left[\begin{array}{l}
\mathbf{L}_{1} \\
\mathbf{L}_{2} \\
\mathbf{L}_{3}
\end{array}\right]=\left[\begin{array}{lll}
I_{1} & 0 & 0 \\
0 & I_{2} & 0 \\
0 & 0 & I_{3}
\end{array}\right]\left[\begin{array}{l}
\omega_{1} \\
\omega_{2} \\
\omega_{3}
\end{array}\right]
$$

Moment of inertia $\left(I_{\mathrm{i}}\right)$ : Resistance to rotational acceleration of an object.

With respect to Figure 1, for a cylindrical rod of mass $m$, length $L$, and radius $a$ grasped at one end at a distance $r$ from the center of mass with the wrist at a distance $d$ from the center of the rod, the moment of inertia about the axis through the wrist and parallel to the $x$-axis is:

$$
I_{1}=\frac{m}{12}\left(3 a^{2}+L^{2}\right)+m\left(r^{2}+d^{2}\right)
$$

If the rod is thin,

$$
I_{1}=\frac{m}{12} L^{2}+m\left(r^{2}+d^{2}\right)
$$

For a cylindrical rod, the moment of inertia $\left(I_{2}\right)$ about the axis through the wrist and parallel to the $z$-axis is the same as $I_{1}$.

The moment of inertia about the axis through the wrist and parallel to the rod is:

$$
I_{3}=m a^{2}+m d^{2} \text { for hollow rods, }
$$

or

$$
=m a^{2} / 2+m d^{2} \text { for solid rods. }
$$

Since $m$ equals $\rho \pi a^{2} L$, where $\rho$ is the density of the rod, $I_{1}$ and $I_{2}$ are proportional to the density of the rod, the diameter squared, and the length to the third power. Simlarly, $I_{3}$ is proportional to the density of the rod and the diameter to the fourth power. 